\title{
Social work students' experiences of using the Egan Skilled Helper model
}

\section{Sally Riggall ${ }^{1}$}

Abstract: This paper aims to assess the value of the Egan Skilled Helper model in helping social workers in training to develop their communication skills with service users. The model was taught to first year BSc Social Work students. During their subsequent first assessed practice placement fifteen volunteers participated in focus groups. Here they discussed how useful the Egan model was as a communication and problem management tool for working with service users. A key finding is that students were able to transfer their skills learning into practice. Further findings are that students improved their communication skills and were better able to place service users at the centre of decision making. The implications of these findings for social work training are discussed together with some of the challenges of working with the model.

Key Words: communication skills; Egan model; service user empowerment; social work; teaching and learning.

1. Senior Lecturer, School of Health and Social Care, Universit of Lincoln

Address for correspondence: School of Health and Social Care, Faculty of Health, Life and Social Sciences, University of Lincoln, Brayford Pool, Lincoln, LN6 7TS. sriggall@lincoln.ac.uk

37 J. of Practice Teaching \& Learning 11(1), pp.37-53. DOI: 10.1921/ 175951511X651940. @ wËb 
Sally Riggall

\section{Introduction}

This paper examines how learning the Egan Skilled Helper model equips BSc Social Work students with effective communication skills which place service users at the centre of the helping process. An additional aim is to find out whether this learning can be transferred into practice.

Helping students to develop effective, empathic communication skills is an established tradition within social work teaching programmes. However, there is little research which explores the process and effect of teaching and learning in terms of communication skills and their theoretical base. Trevithick et al. (2004) undertook for the Social Care Institute for Excellence a knowledge review of teaching and learning of communication skills in social work education. In their review Trevithick et al. found that out of 8023 relevant papers only 16 addressed a theoretical underpinning to communication skills development. No papers were identified that commented on students' experience of different communication models or their preferences. Trevithick et al. (2004) found there was little evidence of evaluation of underpinning knowledge being carried out and even less consideration relating to how successful the transfer of learning is into practice. Trevithick et al. argue that skills learning needs to be based on a proven knowledge base that is relevant to social work and that skills learned should be integrated into practice learning. The Social Work Taskforce Report (2010 p. 21) emphasises the importance of social workers being able to work in a person-centred manner 'to support people to manage their own affairs where possible and to assist in finding solutions which balance choice and control for the individual.'

Social work students commonly and mistakenly believe their role is to tell service users what to do. The Skilled Helper model, developed by Gerard Egan, was chosen for this study because it specifically addresses this values issue. The model helps students to enable service users to take responsibility for their own decision making. The threestage model is complex and cumulative in its development of skills. It emphasises the importance of developing empathic relationships with service users, describes how to encourage people to talk about their lives and to establish exactly what their needs are. Solutions to problems are then sought together. Egan refers throughout his work to 'clients' rather than 'service users' and this paper uses these terms interchangeably.

The overall project has been influenced by a number of papers

38 J. of Practice Teaching \& Learning 11 (1), pp.37-53. DOI: 10.1921/ 175951511X651940. @ w\&-b 
which investigate social workers' use of empathy and the teaching of communication skills. Forrester et al. (2008 p. 41-51) highlighted the importance of empathic responding skills in social work and especially in forming good working relationships with service users and their families. Their findings established that social workers who were skilled in using empathic responses encouraged parents to be much more open about what was happening in their families than social workers who lacked these skills. Forrester et al. commented that most social workers did not use empathy and reflection or summarising skills, favouring instead interventions which consisted in the main of a series of closed questions. They found that questions outnumbered complex empathic reflections by more than fifteen to one. Complex reflections are where information, feelings, behaviours and thoughts from input other than the immediately preceding sentence from the client is reflected back. These were rarely used. Forrester et al. emphasised the importance of raising concerns in a climate which highlights to service users their existing strengths, a factor which is seldom prioritised by social workers. The authors found a relationship between the use of complex reflections and increased client disclosure. They concluded that raising concerns in an empathic manner would increase social workers' likelihood of keeping and maintaining their relationship with both the parent and child.

The importance of empathic responding is also highlighted in a survey of communications skills teaching undertaken by Dinham et al. (2003) who stressed the importance of understanding the problems of service users. The authors also emphasised the essentiality of a coherent knowledge framework to underpin skills teaching and learning. They indicate that the framework should have a well established base of values and principles that encompass service user empowerment, i.e. working with service users to produce agreed outcomes. In their work with children with disabilities, Woodcock and Tregaskis (2008) highlighted the need for social workers in training to develop active listening skills which help parents to express their feelings of anger and frustration in a safe and non-judgemental environment. The authors focused on the finding from Trevithick et al. (2004) which established the centrality of communications skills teaching within the social work degree. Woodcock and Tregaskis agreed with Trevithick that a lack of an underpinning knowledge base when teaching communications skills was a serious omission and if this was addressed, it would enable skills

39 J. of Practice Teaching \& Learning 11(1), pp.37-53. DOI: 10.1921/ 175951511X651940. @ wEb 
to be applied more easily in different settings. This point was also raised by Moss et al. (2007:711) whose research found that 'communication skills have been taught but not reflected upon; experienced but not theorised.'

The Egan Skilled Helper model provides an underpinning framework which provides structure for students in their communication with service users.

Egan (2010) draws from a number of theorists in presenting his model, including Carl Rogers who, as Egan states in an interview with Sugarman (1995), was highly influential. Rogers writes at length about the power of empathic responding and how difficult it is to accomplish. However, this one skill is the key factor that enables the client to grow and change in a climate of understanding or a good attempt at understanding (Rogers, 1961). While Egan draws widely from Rogers's theory in his analysis of the importance of building a non-judgemental relationship with the client in stage one of his model, he nevertheless states clearly in his interview with Sugarman that empathic responding, unconditional positive regard and congruence are not enough to evoke change. As a result of this thinking, Egan developed his Skilled Helper model which emphasises both the relationship and client action. Within the model, stage one skills involve finding out what is currently going on for the client. The client is listened to with empathy; challenging skills are used, again with empathy not only to help the client learn about their own ways of being but also to help them acknowledge and build on their own strengths. In stage two, the preferred picture is identified and goals devised. At all times the client is encouraged to state what they want or need that might help them. Finally, in stage three, the client, with assistance from the helper, develops strategies to achieve their goals. Using the model may enable the student social worker to maintain a client-centred approach by empathically responding to service users, challenging them appropriately and placing them at the centre of decision making. The study which forms the basis for this paper sets out to investigate the way that students make use of this model in practice.

40 J. of Practice Teaching \& Learning 11(1), pp.37-53. DOI: 10.1921/ 175951511X651940. @ wEb 


\section{Study aims and methodology}

This is a small scale study, the aim of which is to assess how valuable the Egan Skilled Helper Model is in helping social workers in training to develop their communication skills. The study primarily explores ways of keeping the service user at the centre of decision making. The study also investigates whether learning was effectively transferred into practice with service users.

The model was taught to two different groups. The first comprised full-time social work students (who had little experience of working with service users) and the second consisted of part-time students on the employment-based course (who had substantial experience and held a case-load). Both groups learned the model within the Communicating and Engaging with Others module.

\section{Methodology: Full-time students}

The model was taught to the full-time students in the term prior to their first practice placement. The module consisted of five full days of workshops, over a five week period, where students learned and practised the model with each other. Six groups, 115 students in total, attended. On the final day of the module, a request was made to the whole group for volunteers to take part in focus group sessions which would be held during their first social work practice placement. The objective was for students to employ the Egan model with service users during their placement and reflect on how the model had helped them to place decision making control with service users.

A limitation of the study is that only five students from this group (three women and two men) subsequently took part in each of four focus groups. All the students were white British (a further limitation), and aged in their twenties and thirties. None of the students had any previous social work experience. In the focus groups, students gave feedback on how useful they had found the model. The sessions lasted between 30 and 60 minutes and were recorded electronically and then transcribed.

Approval for the project was given by the Ethics Committee at the University of Lincoln. The students maintained the confidentiality of service users with whom they worked and no information was used which would have identified any individual.

41 J. of Practice Teaching \& Learning 11 (1), pp.37-53. DOI: 10.1921/ 175951511X651940. @ wEbb 


\section{Methodology: Part-time, employment-based students}

A separate group of ten part-time students taking part in this study were day-released from their workplaces and already held a case load of service users. These students studied and practised the model in a similar way to the full-time students over a period of four weeks for one day per week. A request was made for volunteers to participate in one focus group session one month after the module finished. All ten students (eight women and two men, all white British with an average age of 30) agreed to take part. In the focus group they reflected on their experience of using the model with service users and commented on how it had changed their practice. The session lasted for one hour and was again recorded electronically. Participants in this group had considerably more experience of working with service users than the full-time group.

A distinction is made between the two groups in the presentation of the data. This is because the employment-based students already had an existing relationship with service users and were changing the way they used communication skills. This contrasted with the full-time students who were beginning their first practice placement and thus forming new relationships.

\section{Methodology applicable to both groups}

Participants of both focus groups were asked to comment on the following:

- How had students developed their communication skills as a result of learning the Egan model?

- What impact did using the model have on placing the service user at the centre of the helping process?

- How effective was the transfer of knowledge from the classroom into practice?

An oral method of collecting data as opposed to written feedback was chosen for the study because it placed less pressure on students and at the same time promoted a far more detailed and dynamic response which was in line with the model itself. All of the participants were keen to contribute their experiences to the study.

42 J. of Practice Teaching \& Learning 11 (1), pp.37-53. DOI: 10.1921/ 175951511X651940. @ w\&b 


\section{Findings}

The students who took part in this study were working in a variety of settings, some statutory, some voluntary. In order to maintain confidentiality, quotations from the full-time students are given the prefix ' $F$ ' and the employment-based, part-time students are prefixed with 'P'.

\section{How students developed their communication skills as a result of learning the Egan model}

\section{The importance of having a framework to follow}

The first point raised by the students concerned how the model helped them to develop confidence in their communication skills and understand how their relationship with the service user was progressing. A factor that contributed to developing students' confidence was having a 'map' to follow. Egan (2010) writes about helpers holding the model in their heads as a map so they know where they are going with it and this was echoed by a student from group F who stated:

It's my bible that guides me to be the way I want to be. It's good.

This was also confirmed by another group F student who stated that having a model from which to work was useful:

\footnotetext{
It has definitely helped having a model in your head when doing assessments, when we were first told we would be doing assessments that's the first thing I thought about. It was worth learning.
}

\section{Developing relationships}

The students reported that the Egan model also helped them to gain rapport with service users. As mentioned previously, Egan's humanistic relationship building skills are taken from Carl Rogers's (1961) work where Rogers states that it is the attempt at empathy that develops the relationship rather than getting it right. He states that the client will soon clarify what they really

43 J. of Practice Teaching \& Learning 11(1), pp.37-53. DOI: 10.1921/ 175951511X651940. @ w\&b 
feel and mean if the helper tries to be empathic. The following point from a group F student illustrated his developing skills:

The first time I tried to use an empathic response she replied 'of course it isn't!' So I thought, 'OK, she's put me right'. I know it is about having a go at being empathic though.

As stated, students from the part-time Employment-based BSc in Social Work were more experienced in working with service users and already had their own ways of working. One student from this group demonstrated the effect of learning active listening skills from the Egan model:

I used the model with the whole family. What I found was that they all got a chance, without anybody criticising them, to say what they felt and to just have someone there as a medium to sit and allow them to do that. It really worked for that family.

\section{Developing empathic responding skills}

Gaining rapport with the client by using empathic reflections is an essential skill (Forrester et al., 2008) and an important part of the Egan model. A group P student demonstrated she was able to reflect on using empathic responses:

I worked with a person whose husband had been diagnosed with a terminal illness. It was hard to get through to her. When she had unloaded, I used the empathic response: 'from what you have said to me I think you are really scared and you are really trying to protect your husband but you are worried about what is going to happen? I didn't realise at the time but I said what she was feeling. And she got my hands and kind of cried and that bit, I wasn't actually comfortable with. I think if I hadn't used that response... I don't think I would have been where I was with that lady or her husband and I don't think we would have got things right to be perfectly honest so it was really useful for that but on the other hand it took a bit of, you know ....

The above participant has highlighted the effect of employing Egan's skills of fully engaging with what a client might be thinking and feeling. She demonstrated how unused clients might be to a demonstration of understanding at this level and also how challenging this was for her too. Currer (2007) comments that social workers often find it difficult to

44 J. of Practice Teaching \& Learning 11(1), pp.37-53. DOI: 10.1921/ 175951511X651940. @ wEb 
respond to strong emotional reactions from service users fearing opening the floodgates and causing more harm than good. Assisting students to first anticipate and then work with strong emotional reactions would need to be included in the teaching of the Egan model.

\section{Developing challenging skills}

The model describes how to use challenging skills. However, challenging was not alluded to so frequently by students and it seems this is a more difficult aspect of the model with which to get to grips. Egan (2010) writes at length about this area and states that good, effective challenging is essential in helping clients to manage their situations better. Only one student (from group P) discussed the area of challenging and it is clear that she was hesitant in using this skill:

I think it's about recognising when not to use it as well. I don't know whether it is because I recognised that it was not going to be useful or that I was

frightened of using it.

Although the student demonstrated the ability to be reflective there is clearly a difficulty in using challenging skills. Raising concerns by using challenging skills distinguishes social work from counselling (Forrester et al., 2008) and as such, is an important skill for workers to develop. Assisting students to develop confidence in practising challenging might be a useful development with future groups.

\section{Assisting service users to plan action}

The study indicates that although some students found the first part of the model (active listening skills) the easiest to remember, students who used all three stages of the model experienced this as most beneficial in aiding clients to action. One student from group P who worked with children with profound learning disabilities demonstrated that using the model had had an effect on both the client's action and also on developing the relationship:

I started off using the first stage with children with their parents, talking to them and using empathic responses when they are upset and I've been building a relationship

45 J. of Practice Teaching \& Learning 11(1), pp.37-53. DOI: 10.1921/ 175951511X651940. @ wEbb 
up with a parent to the point where in his review he pointed out he had a good bond with me. We have started with the little things and we are building up to the bigger things, for example getting pictures up on the walls. The little things build onto the bigger things. It just seems to be working really well.

The above point illustrates that students learn from the model to set goals with their service users and that these goals do not have to be huge, small changes are useful in themselves (Egan, 2010).

\section{Debating the usefulness of the model}

One student from group P felt that learning the Egan model had taught him nothing new. He felt that he employed the skills naturally, stating:

There isn't the chance to sit back and empathise, as good and as useful as you would say that would be. We are doing a lot of the Egan model anyway because it is what we do.

The above student already had skills of which he was unaware but according to Koprowska (2010) tutors need to help students to build on communication skills they already possess. The comment above demonstrates that helping students to develop conscious awareness of the difference between skills they bring to the classroom already and new skill development based on theory is a challenge for tutors. The student also highlighted time pressures he was under and how he felt it was too time-consuming to use empathic responses. However, other students commented that time spent using these skills moved the client towards action and resolution more quickly. This will be discussed later in the next section of this paper.

\section{Keeping the service user at the centre of the process}

\section{Empowerment}

The study sought to find out whether using the Egan model helped students to place the service user at the centre of decision making. This was found to be the case with the following group F student:

46 J. of Practice Teaching \& Learning 11(1), pp.37-53. DOI: 10.1921/ 175951511X651940. @ w\&b 
Reflections are much better than asking direct questions, you're prompting but it flows nicely. They are making their own decisions. We're asking them what they would like and the responses are a lot better and we're getting to action quite a lot quicker and they are coming out thinking they've actually done the job. They've had their own power there and made their own decisions and we're just the helpers.

Another student from group F also commented on how the model helped her to place the service user at the centre of the process and the importance of enabling the client to take control and search out a way of managing their problem more successfully:

With one client we looked at where was she at now, and through that we moved on to what does she need or want. She wasn't attending school and it turned out she was worried about what was going on at home when she was at school and felt she couldn't attend all day. We talked about different options and in the end it turned out she does want to stay in school and gain a diploma.

I like the model because they are finding the answer. She was saying where she wanted to be. I felt because she had given a solution she had gone from her head being down coming into the room, not really wanting to engage to being upbeat, open: her complete body language had changed.

The same student illustrated that she can use the Egan model approach of finding out what the client needs rather than telling the service user what to do:

So much was going on in her life that you couldn't really comprehend to be honest, for someone of that age to be going through it, but deep down she did want to stay in education and learnt that there was something out there. I don't want to criticise, but the person who has been working with her has been telling her what she should be doing and telling her if she didn't do that how it was going to affect her. I think that is why she has not engaged.

\section{Resisting problem solving}

Learning the Egan model enabled students to curb their natural tendencies of trying to solve things for the service user and of giving advice. Egan

47 J. of Practice Teaching \& Learning 11 (1), pp.37-53. DOI: 10.1921/ 175951511X651940. @ wE-b 
(2010) writes about power and control in the relationship and how telling the service user what they should be doing embeds this power differential. This is reflected in the student's comments above.

A group F participant demonstrated she could help the client to own their progress and to move towards becoming a more independent decision maker which is a key aspect of the model:

At the end of the day it is their life and if they have achieved that all themselves with you helping them, then they can take all the credit for that and it helps them feel better. And if they feel better and can see the possibilities that they could have and they achieve them themselves: they are more likely to stick to that way of life and it's more positive for them.

Another group F student demonstrated she had absorbed the model into her way of working while at the same time reflecting on her struggle to stay within the client's frame of reference:

I use the model and let them talk but in the back of my head or when I reflect, sometimes I think, 'Oh God, please, just leave the relationship' but you can't say that. It is still there and I've got to do something about that.

The theme highlighted above is one of the barriers which tutors have encountered in teaching the Egan Skilled Helper model, namely, helping students to learn that their role is not to tell the client what to do but to find out what the client wants or needs and from this to establish realistic and achievable goals. Tutors work with students who frequently ask 'But when can we tell the client what to do?' and 'But what if you know what the client ought to do?' Helping students make this leap has been a challenge but one which is fundamental to enabling students to place the service user at the centre of decision making. This is an area that the Egan model addresses clearly by emphasising that the client is the expert on themselves (Egan 2010).

\section{Maintaining the transfer of knowledge from the classroom into practice}

Each of the students demonstrated that they had absorbed and remembered the skills they had learned and that they could employ these skills at a

48 J. of Practice Teaching \& Learning 11 (1), pp.37-53. DOI: 10.1921/ 175951511X651940. @ w\&b 
later date, several months after the module ended. One group P student demonstrated this by referring to stage three forcefield analysis which is a method of helping the client to assess what would help them adhere to their chosen strategy and what might hinder them in this process:

The bit I have used quite a bit is actually the last stage, looking at change and using forcefield analysis and also possibilities where they choose some and what will help them.

Another student from group P demonstrated sustained knowledge by commenting on Egan's (2010) 'SOLER' technique (Sitting squarely, Open body language, Leaning forward, maintaining Eye contact, staying Relaxed) and also the latter stages of the model:

I've used stages two and three in addition to the stage one skills. I'm working with people with learning disability: we use a person-centred approach. I've been helping people look at what they want to do and formulating plans and the best way forward. The model has also had a positive response from colleagues.

Some students demonstrated they developed lasting skills in all three stages of the model. One, an experienced practitioner from group P, talked about how he had adopted the whole model and how this had had an impact on his workplace practices over a period of time:

Managers come from outside saying 'we are going to do this and that' and you say 'no'. It's made you think, 'no, you don't do that: you've got to listen to what they want' so we've put that into practice quite a lot. It has changed working practices. Really, it has. We imposed, or I imposed my own wants, I wanted them to do things, as my own values, 'you should be doing this and going there' and now I step back and find out what they want. I knew what they should be doing and am trying to think differently. It is working and proving to be useful.

The above example shows how this student's values have been influenced by the model. His analysis shows that this way of working is of value to both himself as a helper and to the service user. His comments also illustrate that a change in approach has been adopted and maintained by him in the workplace.

More research into transferring skills from the classroom into the workplace is planned. Students who took part in this study will be

49 J. of Practice Teaching \& Learning 11(1), pp.37-53. DOI: 10.1921/ 175951511X651940. @ wEb 
interviewed in their second social work practice placement to establish whether their new knowledge and skills have been maintained. Engaging interested practice educators by teaching them the model is also planned. Including practice educators would enable students to discuss their progress with the model during supervision. Findings from this planned project will be discussed in a future paper.

\section{Discussion and Conclusion}

The majority of the fifteen students who took part in the study commented that using Egan's Skilled Helper model improved their communication skills. The students particularly liked the framework offered by the model of 'where is the client now, where do they want or need to be and how are they going to get there.' Having this 'map' gave them confidence and actual, practical ways of working collaboratively with service users and placing them in charge of their own lives. Many students early in their training struggle with the temptation to tell service users what to do and it is encouraging that in this study participants found that using the Egan model curbed these tendencies. Instead they worked collaboratively with clients to help them find ways of managing their problems. Consequently they found there was a move towards client self-determination more quickly. The importance of such autonomy has been highlighted by a number of writers including Cambridge (2004) and Cooper and Spencer-Dawe (2006). A key finding of the study is that placing service users at the centre of decision making promotes earlier resolution of issues. A further study will find out whether using the Egan model enables cases to be closed more quickly.

Almost all of the study participants stated that using new skills contained in the Egan model, such as empathic responding, has enabled them to engage at a deeper level. Indeed, Forrester et al. (2008) and the DCSF's (2010) recommendations were that advanced empathy was a vital skill to learn and should be taught in communication skills modules. One project participant, however, described her use of an empathic reflection and then spoke of her subsequent discomfiture at the intense emotional reaction from the service user. Therefore, a point to note is that in teaching the Egan model, students need to be alerted to the possibility that using empathy can be overwhelming, both for themselves and the service user. Training also needs to include skills for working with strongly expressed emotions.

50 J. of Practice Teaching \& Learning 11(1), pp.37-53. DOI: 10.1921/ 175951511X651940. @ w\&-b 
The participants' discussion of how they used the Egan model demonstrates how they have transferred their knowledge from the classroom into practice settings. The five full-time students continued to give feedback on the model throughout their first practice placement demonstrating that their learning from year one was sustained into practice. The feedback from a whole group of ten, employment-based, part-time students has also been useful in evaluating how knowledge has been transferred because these students were already experienced practitioners and held a case-load of service users. A key finding is that these participants have been willing to change their approach by putting into practice a new model. A further study is planned to establish whether their new skills have been sustained over a longer period.

The study has limitations, mainly concerning its size which was governed inevitably by the availability of students and their capacity to give time to the project. The sample size was small and consisted of exclusively white students, the majority of whom were female. Ashencaen Crabtree and Baba (2001) comment that all Western counselling approaches extoll the importance of individual needs and goals. They argue that in some cultures, such as Islam, the needs of community outweigh those of self-interest and that practitioners need to recognise such factors. Egan comments that his theory may well not fit other cultures, or may need adaptation. However, he also emphasises the importance of recognising diversity within cultures, stating 'clients are individuals, not subcultures or groups' and that practitioners need to use interventions which 'factor in key cultural and personal-culture variables' (Egan, 2010 pp.51-52). Working with these corollaries can be highly challenging for practitioners and since there is little research in this area, further exploration would be useful.

Another limitation is that the interest in the subject by the author (who also taught the module) may have influenced the members of the focus group to give more positive feedback than they would have given to an independent third party (Robson, 2002). This constraint will be addressed in the next study by the inclusion of an independent interviewer.

A criticism of the Egan model is that it assumes service users will be able to communicate verbally. Indeed, Rowland and McDonald (2009) in their paper on communication skills with service users who have aphasia (people who cannot form words but who have cognitive understanding) raise the issue of how social work students are taught to communicate with people who do not have verbal skills. The authors emphasise the importance of enabling all service users to be able to express their needs and voice their

51 J. of Practice Teaching \& Learning 11(1), pp.37-53. DOI: 10.1921/ 175951511X651940. @ wEbb 
concerns, often non-verbally. They also emphasise the crucial importance in these instances of helpers using empathic responses. The Egan model places the service user at the centre of decision making by encouraging the helper to first find out their needs and then collaborate in establishing achievable goals. This process does not have to consist of using purely verbal skills. More creative approaches can be employed such as using symbols, artwork and advocacy skills. One student in the study reported using the Egan model when working with children with profound learning disabilities to establish what they needed or wanted and this can be taken as a starting point to a more detailed investigation in the future.

A planned development from the current project is to involve the same group of students in assessing further the efficacy of the model including whether it leads to cases being closed more quickly. It will also examine whether students' knowledge and skills from learning the model have been sustained over time. A second planned project will find out whether learning could be further embedded by involving practice educators. Trevithick et al (2004) in their report for SCIE suggested social work educators should embed communication skills into practice by working with practice educators and service users in order to maximise student learning within practice itself. Practice educators will have the opportunity to learn the model so that they will be enabled to support the new sample of students who have volunteered to give feedback on the Egan model during their placement. These projects will explore further the usefulness of the Skilled Helper model in enabling students to develop their communication skills.

\section{References}

Ashencaen Crabtree, S. and Baba, I. (2001) Islamic perspectives in social work education: Implications for teaching and practice. Social Work Education, 20, 4, 469-481

Cambridge, P. (2004) Good enough decision making? Improving decision-making in adult protection. Social Work Education, 23, 6, 711-729

Cooper, H. and Spencer-Dawe, E. (2006) Involving service users in interprofessional education: Narrowing the gap between theory and practice. Journal of Interprofessional Care, 20, 6, 603-617

Currer, C. (2007) Loss and Social Work. Exeter: Learning Matters

Department for Children, Schools and Families (2010) Social Work Task Force:

52 J. of Practice Teaching \& Learning 11 (1), pp.37-53. DOI: 10.1921/ 175951511X651940. @ w\&-b 
Building a Safe and Confident Future: Implementing the Recommendations of the Social Work Task Force, [Online], DCFS, London. Available from: http:// publications.education.gov.uk/eOrderingDownload/00306-2010DOM-EN.pdf

Dinham, A. (2006) A review of practice of teaching and learning of communication skills in social work education in England. Social Work Education, 25, 8, 838-850

Egan, G. (2010) The Skilled Helper: A problem management and opportunity development approach to helping. 9th edition. Pacific Grove, CA: Brooks/Cole

Forrester, D., Kershaw, S., Moss, H. and Hughes, L. (2008) Communication skills in child protection: How do social workers talk to parents? Child and Family Social Work, 13, 1, 41-51

Koprowska, J. (2010) Communication and Interpersonal Skills in Social Work. (3rd ed.) Exeter: Learning Matters

Moss, B.R., Dunkerly, M., Price, B., Sullivan, W., Reynolds, M. and Yates, B. (2007) Skills laboratories and the new social work degree: One small step towards best practice? Service users' and carers' perspectives. Social Work Education, 26, 7, 708-722

Robson, C. (2002) Real World Research. $2^{\text {nd }}$ edition. Oxford: Blackwell Publishing Rogers, C. (1961) On Becoming a Person: A therapist's view of psychotherapy. London: Constable

Rowland, A. and McDonald, L. (2009) Evaluation of social work communication skills to allow people with aphasia to be part of the decision making process in healthcare. Social Work Education, 28, 2, 128-144

Sugarman, L. (1995) Action man: An interview with Gerard Egan. British Journal of Guidance and Counselling, 23, 2, 275-287

Trevithick, P., Richards, S., Ruch, G. and Moss, B. (2004) Knowledge Review 6: Teaching and Learning Communication Skills in Social Work Education. [Online], Social Care Institute for Excellence: London. Available from: http://www.scie. org.uk/publications/knowledgereviews/kr06.asp

Woodcock, J. and Tregaskis, C. (2008) Understanding structural and communication barriers to ordinary family life for families with disabled children: A combined social work and social model of disability analysis. British Journal of Social Work, 38, 1, 55-71 